\title{
Microscopic Coexistence of Superconductivity and Magnetism in $\mathrm{Ba}_{1-x} \mathrm{~K}_{x} \mathrm{Fe}_{2} \mathrm{As}_{2}$
}

\author{
Erwin Wiesenmayer, ${ }^{1}$ Hubertus Luetkens, ${ }^{2}$ Gwendolyne Pascua, ${ }^{2}$ Rustem Khasanov, ${ }^{2}$ Alex Amato, ${ }^{2}$ Heidi Potts, ${ }^{3}$ \\ Benjamin Banusch, ${ }^{3}$ Hans-Henning Klauss, ${ }^{4}$ and Dirk Johrendt ${ }^{1, *}$ \\ ${ }^{1}$ Department Chemie, Ludwig-Maximilians-Universität München, D-81377 München, Germany \\ ${ }^{2}$ Labor für Myonenspinspektroskopie, Paul Scherrer Institute, CH-5232 Villigen PSI, Switzerland \\ ${ }^{3}$ Swiss Nanoscience Institute (SNI), Universität Basel, CH-4056 Basel, Switzerland \\ ${ }^{4}$ Institut für Festkörperphysik, TU Dresden, D-01069 Dresden, Germany
}

(Received 22 August 2011; published 29 November 2011)

\begin{abstract}
It is widely believed that, in contrast to its electron-doped counterparts, the hole-doped compound $\mathrm{Ba}_{1-x} \mathrm{~K}_{x} \mathrm{Fe}_{2} \mathrm{As}_{2}$ exhibits a mesoscopic phase separation of magnetism and superconductivity in the underdoped region of the phase diagram. Here, we report a combined high-resolution x-ray powder diffraction and volume-sensitive muon spin rotation study of $\mathrm{Ba}_{1-x} \mathrm{~K}_{x} \mathrm{Fe}_{2} \mathrm{As}_{2}$ showing that this paradigm does not hold true in the underdoped region of the phase diagram $(0 \leq x \leq 0.25)$. Instead we find a microscopic coexistence of the two forms of order. A competition of magnetism and superconductivity is evident from a significant reduction of the magnetic moment and a concomitant decrease of the magnetoelastically coupled orthorhombic lattice distortion below the superconducting phase transition.
\end{abstract}

DOI: 10.1103/PhysRevLett.107.237001

PACS numbers: 74.70.Xa, 61.05.C-, 74.62.Dh, 76.75.+i

The interplay of structural, magnetic and superconducting order parameters is one of the most intriguing aspects in iron based superconductors. In the LaFeAsO (1111) and $\mathrm{BaFe}_{2} \mathrm{As}_{2}$ (122) families, superconductivity (SC) evolves from nonsuperconducting parent compounds with tetragonal crystal structures that are subject to tiny orthorhombic lattice distortions below certain temperatures $\left(T_{s}\right)$. Static long-range antiferromagnetic (AF) ordering emerges at Néel temperatures $\left(T_{N}\right)$ well below $T_{s}$ in LaFeAsO [1], but very close to $T_{s}$ in $\mathrm{BaFe}_{2} \mathrm{As}_{2}$ [2]. The structural and magnetic transitions of the parent compounds are suppressed and finally eliminated by doping of the FeAs layers by electrons or holes, and superconductivity emerges at certain doping levels [3]. With respect to the origin of unconventional superconductivity, the possible coexistence of magnetic and superconducting phases in the underdoped areas of the phase diagrams is of considerable interest. But the coupling of structural, magnetic and superconducting order parameters relies on microscopic phase coexistence that is often difficult to distinguish from mesoscopic phase separation. In the 122-family, microscopic coexistence of these orders is generally accepted for the electron-doped compounds $\mathrm{Ba}\left(\mathrm{Fe}_{1-x} \mathrm{Co}_{x}\right)_{2} \mathrm{As}_{2}$, while conflicting reports exist for the hole-doped compounds $\mathrm{Ba}_{1-x} \mathrm{~K}_{x} \mathrm{Fe}_{2} \mathrm{As}_{2}$.

The coexistence of the orthorhombic structure with SC has first been suggested for $\mathrm{Ba}_{1-x} \mathrm{~K}_{x} \mathrm{Fe}_{2} \mathrm{As}_{2}$ up to $x \approx 0.2$ by $\mathrm{x}$-ray powder diffraction [4], while neutron diffraction experiments additionally showed long-range AF ordering up to $x \approx 0.3$ [5]. Diffraction methods however only provide the mean structural information on a rather long spatial scale, and cannot supply conclusive information

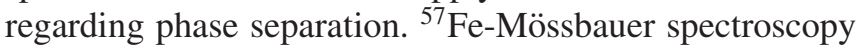
as a local probe indicated microscopic coexistence [6], but other local probes such as $\mu$ SR [7-9] and NMR [10] showed phase separation with nonmagnetic superconducting volume fractions between 25 and $40 \%$. These experiments with almost optimally doped single crystals grown from fluxes constituted the paradigm of phase separation in underdoped $\mathrm{Ba}_{1-x} \mathrm{~K}_{x} \mathrm{Fe}_{2} \mathrm{As}_{2}$.

In contrast to these scattered results, studies with cobaltdoped $\mathrm{Ba}\left(\mathrm{Fe}_{1-x} \mathrm{Co}_{x}\right)_{2} \mathrm{As}_{2}$ yielded convincing evidence for microscopic coexistence [11]. Moreover, competing order parameters became obvious by the concomitant reduction of the orthorhombic lattice distortion $\delta=\frac{a-b}{a+b}$ and magnetic moment $\mu_{\mathrm{Fe}}$ when crossing the critical temperature [12]. This microscopic coexistence supports $s^{ \pm}$symmetry of the superconducting order parameter $[13,14]$ and gives strong evidence for unconventional superconductivity in iron arsenides.

Considering this generally accepted situation for $\mathrm{Ba}\left(\mathrm{Fe}_{1-x} \mathrm{Co}_{x}\right)_{2} \mathrm{As}_{2}$, it is particularly important to clarify the intrinsic behavior of $\mathrm{Ba}_{1-x} \mathrm{~K}_{x} \mathrm{Fe}_{2} \mathrm{As}_{2}$, also because cobalt doping causes additional disorder in the $\left(\mathrm{Fe}_{1-x} \mathrm{Co}_{x}\right)$ As layers, while potassium doping hardly affects the FeAs layers. Thus, if both orders coexist microscopically in $\mathrm{Ba}_{1-x} \mathrm{~K}_{x} \mathrm{Fe}_{2} \mathrm{As}_{2}$, we rather observe the behavior of the clean superconducting FeAs layer. Indeed, a recent neutron diffraction study with polycrystalline material supports early suggestions about microscopic coexistence [15], but gives no conclusive proof, because elastic neutron scattering as a bulk probe is principally unable to distinct whether the magnetic volume fraction or the magnetic moment at the iron site decreases.

In this Letter, we report a combined high-resolution $\mathrm{x}$-ray diffraction and muon spin rotation $(\mu \mathrm{SR})$ study with underdoped $\mathrm{Ba}_{1-x} \mathrm{~K}_{x} \mathrm{Fe}_{2} \mathrm{As}_{2} \quad(x=0,0.19,0.23$, 0.25 ). We unambiguously show the microscopic coexistence of the superconducting and antiferromagnetic 
phase and the competition of the respective order parameters.

Polycrystalline samples of $\mathrm{Ba}_{1-x} \mathrm{~K}_{x} \mathrm{Fe}_{2} \mathrm{As}_{2}$ were synthesized by heating stoichiometric mixtures of the elements (purities $>99.9 \%$ ) in alumina crucibles sealed in silica tubes under purified argon. Potassium evaporation was minimized by using alumina inlays. The mixtures were heated to $873 \mathrm{~K}(50 \mathrm{~K} / \mathrm{h})$ for $15 \mathrm{~h}$. The products were homogenized in the crucible and annealed at $923 \mathrm{~K}$ for $15 \mathrm{~h}$. Finally the powders were cold pressed into pellets, sintered for $20 \mathrm{~h}$ at $1023 \mathrm{~K}$ and cooled to room temperature by switching off the furnaces. Lattice parameters were obtained by temperature-dependent X-ray powder diffraction ( $\mathrm{Co}, \mathrm{Cu}, \mathrm{Mo}-K_{\alpha 1}$ radiation) and Rietveld refinements using the TOPAS package [16]. Figure 1(a) shows a typical XRD pattern. Only traces of $\mathrm{AsO}_{2}$ and weak magnetic FeAs impurity phases were detected, which neither disturb the local probe $\mu$ SR [17] nor the susceptibility and $\mathrm{XRD}$ measurements. Ba:K ratios were examined by refinement of the site occupancy parameters and cross checked by ICP-AAS chemical analysis. $\mu$ SR measurements have been performed using the GPS and DOLLY spectrometers located at the $\pi M 3$ and $\pi E 1$ beam lines of the Swiss Muon Source at the Paul Scherrer Institut, Switzerland. The data have been analyzed using the MUSRFIT package [18].

$\mathrm{X}$-ray powder patterns of the samples revealed the known structural phase transitions from tetragonal to orthorhombic symmetry. In agreement with our earlier studies [4], also Ref. [15] showed that the orthorhombic distortion depends on the potassium concentration and is finally absent if $x \geq 0.3$. Figure 1(b) shows the temperature dependency of the (112) reflections. While the clear splitting, or at least broadening of the peak is visible at $x=0.19$ and 0.23 , it is apparently absent at $x=0.25$. However, a closer inspection reveals the onset of peak broadening below $\approx 70 \mathrm{~K}$ also in this case. From this we obtained the tetragonal to orthorhombic transition temperatures $T_{s}=140,98,84$, and $70 \mathrm{~K}$ for $x=0,0.19$, 0.23 , and 0.25 , respectively. The lattice parameters obtained from Rietveld-refinements are shown in Fig. 1(c). The continuous splitting indicate that the transitions are likely second order at least in the doped samples, even this has also been suggested for the undoped parent compound $[2,19]$. It is obvious that potassium doping of $\mathrm{BaFe}_{2} \mathrm{As}_{2}$ reduces the transition temperature $T_{s}$ and also the extent of the lattice parameter splitting, which is still visible at $x=0.25$ where $T_{c}$ is already $32.6 \mathrm{~K}$.

The main goal of this study is to clarify how magnetism and superconductivity coexist in the underdoped region of the $\mathrm{Ba}_{1-x} \mathrm{~K}_{x} \mathrm{Fe}_{2} \mathrm{As}_{2}$ phase diagram. For this reason $\mathrm{x}$-ray, ac susceptibility, and $\mu$ SR measurements have been performed on the very same samples to investigate their superconducting and magnetic properties, respectively.

The ac susceptibility of finely ground powder samples were measured between 3.4 and $45 \mathrm{~K}$ at 8 Oe and $1.333 \mathrm{kHz}$. Diamagnetic signals were detected below $T_{c}=22.7 \quad(x=0.19), 28.5 \quad(x=0.23)$, and $32.6 \mathrm{~K}$ $(x=0.25)$ as shown in Fig. 2(a). The superconducting volume fractions of all samples are close to $100 \%$ and prove bulk superconductivity.

Muon spin rotation measurements in a weak transverse field $(w \mathrm{TF}-\mu \mathrm{SR})$ provide an easy means to measure the magnetic volume fraction. In Fig. 2(c) the magnetic volume fractions obtained by such measurements in $B_{\text {ext }}=50$ Oe are shown for various $\mathrm{Ba}_{1-x} \mathrm{~K}_{x} \mathrm{Fe}_{2} \mathrm{As}_{2}$ samples $(x=0.0,0.19$, and 0.23$)$ as a function of temperature. a)

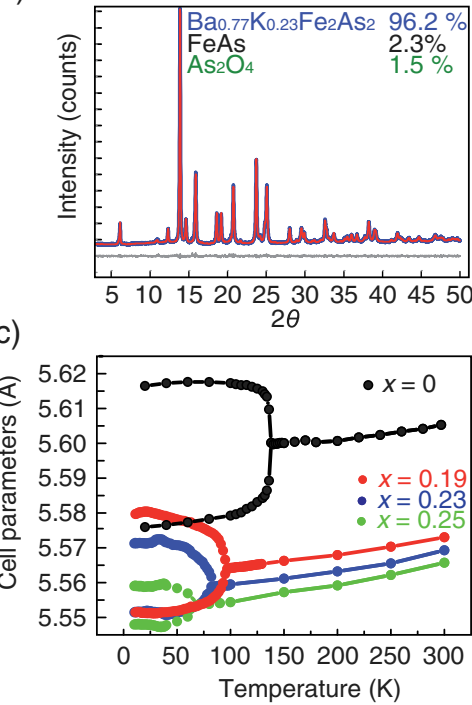

b)

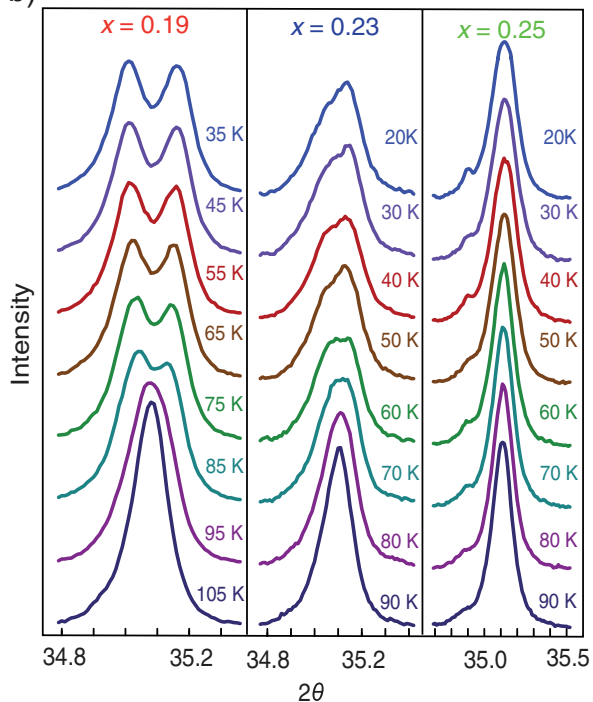

FIG. 1 (color online). (a) X-ray powder diffraction pattern (blue) and Rietveld fit (red) of $\mathrm{Ba}_{1-x} \mathrm{~K}_{x} \mathrm{Fe}_{2} \mathrm{As}_{2} \quad(x=0.23$ ). (b) Temperature dependence of the (112) reflections of for $x=0.19,0.23$, and 0.25 . (c) $a$ - and $b$-axis cell parameters as a function of temperature. 

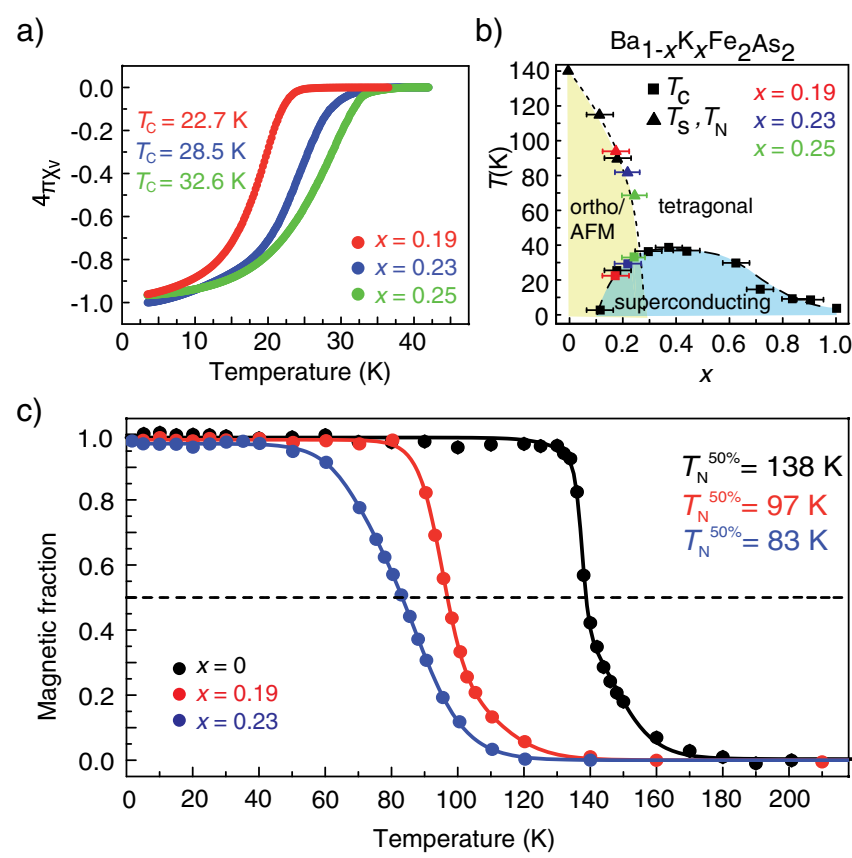

FIG. 2 (color online). (a) Magnetic susceptibility measurement of $\mathrm{Ba}_{1-x} \mathrm{~K}_{x} \mathrm{Fe}_{2} \mathrm{As}_{2}$ with $x=0.19,0.23$, and 0.25 showing $\approx 100 \%$ diamagnetic shielding. (b) Structural, magnetic and superconducting phase diagram of $\mathrm{Ba}_{1-x} \mathrm{~K}_{x} \mathrm{Fe}_{2} \mathrm{As}_{2}$. (c) Magnetic volume fraction as a function of temperature obtained from $w \mathrm{TF}-\mu \mathrm{SR}$ measurements.

For all samples a transition to a magnetic state is observed. From this the magnetic transition temperature where $50 \%$ of the volume is magnetic has been determined to $T_{\mathrm{N}}=138,97$, and $83 \mathrm{~K}$ for the three samples, which are very close to the structural transition temperatures $T_{s}$. The magnetic volume fraction reaches $100 \%$ for all three samples and, most remarkable, does not change below the superconducting $T_{c}$. Therefore, these results, together with the $100 \%$ superconducting shielding signal observed in the ac susceptibility measurements, prove the microscopic coexistence of magnetism and superconductivity in the orthorhombic phase of our samples. The structural, magnetic and superconducting transition temperatures are compiled in the phase diagram depicted in Fig. 2(b).

The orthorhombic distortion in terms of the structural order parameter $\delta$ is shown in Fig. 3(a). In the $x=0.19$ sample, $\delta$ achieves a clear maximum $\delta_{\max } \approx 27 \times 10^{-4}$ at the superconducting transition temperature close to $23 \mathrm{~K}$ and then decreases to lower temperatures. Higher potassium concentrations further decrease $T_{s}$ to $84 \mathrm{~K}$ while $\delta_{\max } \approx 20 \times 10^{-4}$ again coincides with $T_{c}$ at $28.5 \mathrm{~K}$ $(x=0.23)$. This trend continues at $x=0.25$ with $T_{s} \approx$ $70 \mathrm{~K}, T_{c}=32.6 \mathrm{~K}$ and $\delta_{\max } \approx 13 \times 10^{-4}$. This behavior is similar to $\mathrm{Ba}\left(\mathrm{Fe}_{1-x} \mathrm{Co}_{x}\right)_{2} \mathrm{As}_{2}$ [12]; however, we do not observe the further linear decrease of $\delta$ at lower temperatures back to a quasitetragonal structure, but rather saturation of $\delta$. Also in contrast to the Co doped material, we find that the effect becomes smaller with increasing

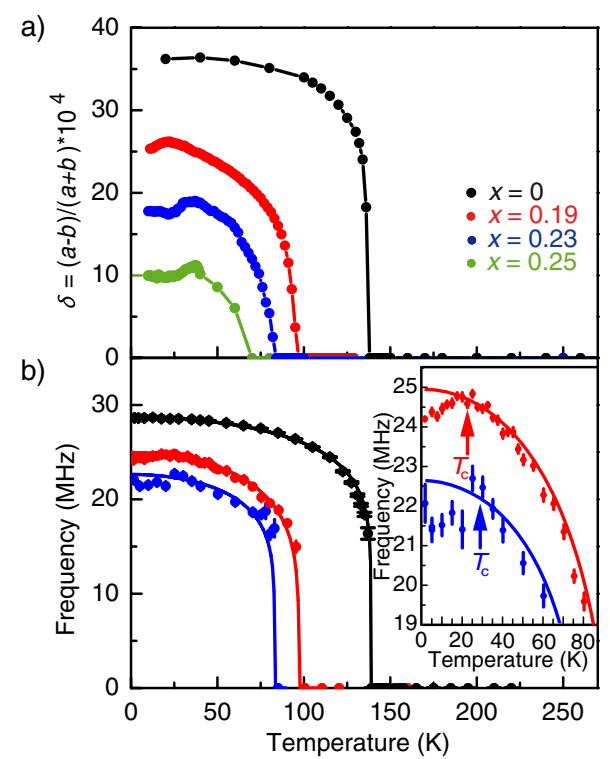

FIG. 3 (color online). Orthorhombicity parameter $\delta$ and $\mathrm{ZF}-\mu \mathrm{SR}$ frequency (magnetic order parameter) of $\mathrm{Ba}_{1-x} \mathrm{~K}_{x} \mathrm{Fe}_{2} \mathrm{As}_{2}$ as a function of temperature.

potassium concentrations $x$. The reason for that is not yet clear. We suggest that the stronger effect in the case of Co doping may be connected with the fact, that magnetic ordering is weakened not only by the electron doping, but additionally by the disorder that is introduced by the cobalt atoms at the iron sites. Thus the competition of superconductivity and antiferromagnetism for the same electrons may affect the $\left(\mathrm{Fe}_{1-x} \mathrm{Co}_{x}\right)$ As layers more efficiently than the clean FeAs layers in the $\mathrm{K}$ doped material.

To elucidate further the magnetic properties of $\mathrm{Ba}_{1-x} \mathrm{~K}_{x} \mathrm{Fe}_{2} \mathrm{As}_{2}$, zero field (ZF) $\mu \mathrm{SR}$ measurements have been performed. The ZF $-\mu$ SR spectra shown in Fig. 4 exhibit well defined muon spin precessions below $T_{\mathrm{N}}$. The increased damping of the precession at higher doping indicates a decreased level of magnetic homogeneity throughout the sample. Anyhow, the observation of a spontaneous $\mu$ SR precession is the signature of a longrange magnetically ordered phase even at $x=0.23$.

As already observed in other $\mathrm{Fe}$ based superconductors [8,20-22] the ZF spectra are composed of two distinct precession frequencies, which is clearly visible from the beating of the time domain data in Fig. 4. This has been interpreted as two magnetically inequivalent muon stopping sites in the structure. The data can be well fitted with two damped cosine functions and a nonrelaxing longitudinal tail with an amplitude of $1 / 3$. This is the fingerprint of a commensurate magnetic structure which is static on the time scale of $\mu$ SR [23]. Note that in underdoped samples of the related $\mathrm{Ba}\left(\mathrm{Fe}_{1-x} \mathrm{Co}_{x}\right)_{2} \mathrm{As}_{2}$ family only a strongly overdamped oscillation can be observed [24,25]. This indicates that the doping in the Ba layer causes considerably less disorder into the magnetic system. Another difference is that in the Co-doped systems $\mu \mathrm{SR}$ spectra consistent 


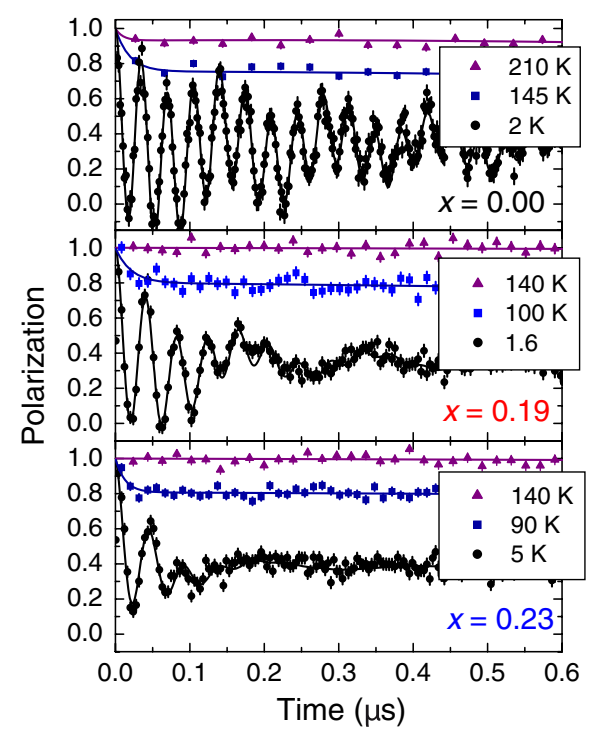

FIG. 4 (color online). Zero field $\mu$ SR spectra for characteristic temperatures (above, at, and below the transition) for $\mathrm{Ba}_{1-x} \mathrm{~K}_{x} \mathrm{Fe}_{2} \mathrm{As}_{2}$ powder samples with $x=0.0$, 0.19, and 0.23.

with incommensurate order have been found. ZF- $\mu$ SR allows us to precisely determine the temperature dependence of the magnetic order parameter (Fe moment) which is proportional to the measured $\mu \mathrm{SR}$ frequency. The higher of the two $\mu$ SR frequencies is shown in Fig. 3(b) together with the orthorhombicity parameter $\delta=\frac{a-b}{a+b}$ deduced from the XRD measurements.

The magnetic order parameter shown in Fig. 3(b) decreases alike the orthorhombicity as a function of potassium doping. Most remarkable, however, is the decrease of the magnetic order parameter (Fe moment) below the superconducting $T_{c}$ clearly visible in the inset of Fig. 3(b). Here we would like to mention that $\mu \mathrm{SR}$ as a local probe is able to measure the magnetic volume fraction [see Fig. 2(c)] and the size of the ordered moment (via the ZF $-\mu$ SR frequency) separately unlike it is done in scattering experiments where the product of both quantities is measured. Taking all data together it is obvious that all investigated samples remain $100 \%$ magnetic, but that the ordered Fe magnetic moment as well as the orthorhombicity decrease below the superconducting $T_{c}$. In other words, superconductivity and magnetism coexist on a microscopic scale, but compete for the same electrons in the underdoped region of the $\mathrm{Ba}_{1-x} \mathrm{~K}_{x} \mathrm{Fe}_{2} \mathrm{As}_{2}$ phase diagram.

In summary, our results prove the paradigm of phase separation in $\mathrm{Ba}_{1-x} \mathrm{~K}_{x} \mathrm{Fe}_{2} \mathrm{As}_{2}$ wrong. Instead we find compelling evidence of microscopic coexistence of superconductivity with magnetic ordering from combined x-ray and $\mu \mathrm{SR}$ data. The competition for the same electrons reduces the magnetic moment below $T_{c}$, while the magnetic fraction remains $100 \%$ according to volume-sensitive $\mu \mathrm{SR}$ measurements. The response of the structural and magnetic order parameters at $T_{c}$ is weaker than in Co doped $\mathrm{Ba}\left(\mathrm{Fe}_{1-x} \mathrm{Co}_{x}\right)_{2} \mathrm{As}_{2}$. Since $\mathrm{K}$ doping introduces no disorder in the superconducting FeAs layer, we suggest that we rather observe its intrinsic behavior.

Thanks to Marianne Rotter for the low temperature x-ray diffraction measurements and to Marcus Tegel for support with Rietveld refinements. This work was financially supported by the German Research Foundation (DFG) within the priority program SPP1458, project No. JO257/6-1. Part of this work has been performed at the Swiss Muon Source at the Paul Scherrer Institut, Switzerland.

*johrendt@lmu.de; hubertus.luetkens@psi.ch

[1] C. de la Cruz et al., Nature (London) 453, 899 (2008).

[2] M. Rotter, M. Tegel, I. Schellenberg, W. Hermes, R. Pöttgen, and D. Johrendt, Phys. Rev. B 78, 020503 (2008).

[3] V. Zinth, T. Dellmann, H.-H. Klauss, and D. Johrendt, Angew. Chem., Int. Ed. 50, 7919 (2011).

[4] M. Rotter, M. Pangerl, M. Tegel, and D. Johrendt, Angew. Chem., Int. Ed. 47, 7949 (2008).

[5] H. Chen et al., Europhys. Lett. 85, 17006 (2009).

[6] M. Rotter, M. Tegel, I. Schellenberg, F. M. Schappacher, R. Pöttgen, J. Deisenhofer, A. Gunther, F. Schrettle, A. Loidl, and D. Johrendt, New J. Phys. 11, 025014 (2009).

[7] A. A. Aczel et al., Phys. Rev. B 78, 214503 (2008).

[8] T. Goko et al., Phys. Rev. B 80, 024508 (2009).

[9] J. T. Park et al., Phys. Rev. Lett. 102, 117006 (2009).

[10] M. H. Julien, H. Mayaffre, M. Horvatic, C. Berthier, X. D. Zhang, W. Wu, G. F. Chen, N.L. Wang, and J. L. Luo, Europhys. Lett. 87, 37001 (2009).

[11] D. K. Pratt, W. Tian, A. Kreyssig, J. L. Zarestky, S. Nandi, N. Ni, S. L. Bud'ko, P. C. Canfield, A. I. Goldman, and R. J. McQueeney, Phys. Rev. Lett. 103, 087001 (2009).

[12] S. Nandi et al., Phys. Rev. Lett. 104, 057006 (2010).

[13] R. M. Fernandes et al., Phys. Rev. B 81, 140501 (2010).

[14] R. M. Fernandes and J. Schmalian, Phys. Rev. B 82, 014521 (2010).

[15] S. Avci et al., Phys. Rev. B 83, 172503 (2011).

[16] R. W. Cheary, A. A. Coelho, and J. P. Cline, J. Res. Natl. Inst. Stand. Technol. 109, 1 (2004).

[17] P. J. Baker, H. J. Lewtas, S. J. Blundell, T. Lancaster, F. L. Pratt, D. R. Parker, M. J. Pitcher, and S. J. Clarke, Phys. Rev. B 78, 212501 (2008).

[18] A. Suter and B. M. Wojek, Physics Procedia (to be published).

[19] S. D. Wilson, Z. Yamani, C. R. Rotundu, B. Freelon, E. Bourret-Courchesne, and R. J. Birgeneau, Phys. Rev. B 79, 184519 (2009).

[20] H.-H. Klauss et al., Phys. Rev. Lett. 101, 077005 (2008).

[21] A. Jesche et al., Phys. Rev. B 78, 180504 (2008).

[22] H. Maeter et al., Phys. Rev. B 80, 094524 (2009).

[23] A. Yaouanc and P.D. de Réotier, Muon Spin Rotation, Relaxation and Resonance (Oxford University Press, Oxford, 2011).

[24] C. Bernhard et al., New J. Phys. 11, 055050 (2009).

[25] P. Marsik et al., Phys. Rev. Lett. 105, 057001 (2010). 